\title{
Ingestions Alimentaires Et Performances De Production Et De Reproduction Des Lapines (Oryctolagus Cuniculus) Gestantes Nourries Avec Des Aliments Contenant Des Feuilles De Neem (Azadirachta Indica)
}

Soumanou Toleba S., (PhD)

Faculté des Sciences Agronomiques/

Université d'Abomey-Calavi (FSA/UAC), BENIN

Mahamadou Dahouda, (PhD)

Sévérin D. Vidjannagni, (M.Sc)

Faculté des Sciences Agronomiques/

Université d'Abomey-Calavi (FSA/UAC), BENIN

Centre Technique d'Aviculture et d'élevage des petits animaux

(CETAvi-Bénin), BENIN

$$
\text { Haziz Sina, (PhD) }
$$

Laboratoire de Biologie et de Typage Moléculaire en Microbiologie,

Faculté des Sciences et Techniques/Université d'Abomey-Calavi,

05 BP 1604 Cotonou, BENIN.

Ulrich Natonnagnon, (M.Sc)

Aminatou Harouna, (M.Sc)

Université Africaine de Technologie et de Management

(UATM-GASA Formation), BENIN

Adam D.Adenile (PhD)

Nadine S. I. Anagonou, (PhD)

Faculté des Sciences Agronomiques/

Université d'Abomey-Calavi (FSA/UAC), BENIN

Lamine Baba-Moussa (PhD)

Laboratoire de Biologie et de Typage Moléculaire en Microbiologie, Faculté des Sciences et Techniques/Université d'Abomey-Calavi,

05 BP 1604 Cotonou, BENIN.

doi: 10.19044/esj.2017.v13n15p82 $\quad$ URL:http://dx.doi.org/10.19044/esj.2017.v13n15p82

Abstract

This study was conducted as part of a series of experiments on the use of dried leaves for the production of complete rabbit feed. For this 
purpose, 120 female rabbits, distributed in 4 batches (N0, N5, N10 and N15) with the same number (30) and homogeneous average weights (2595 g to $2708.7 \mathrm{~g}$ ) were followed before and during pregnancy. Each of the 4 lot were composed, in the same proportion, of nulliparous and multiparous females. Those animals were subjected to four Azadirachta indica leaves based foods in different proportions: N0: 0\%; N5: 5\%; N10: 10\% and N15: 15\%. Our data shows that the gestation rates were as follows for the four lots: N0 (90\%), N5 (80\%) and N10 (90\%) and N15 (76.67\%). Except a single female from lot N10, all females were pregnant and gave birth. No significant difference (P> 0.05) was found between the mean of total daily consumptions among the pregnant rabbits of the four batches that ranged from 169.40 to $198.31 \mathrm{~g} / \mathrm{d}$. The average litters of the batches N0 (5.33 rabbits / mother), N5 (6.5 rabbits / mother) and N10 (5.61 rabbits / mother) were similar $(\mathrm{P}>0.05)$ but higher $(\mathrm{P}<0.05)$ than those of lot N15 (4.46 rabbits / mother). At birth, the lot N15 (68.00g) young rabbits' mean weight of was significantly higher $(\mathrm{P}<0.05)$ than those of N5 $(48.63 \mathrm{~g})$ and N10 $(53.62 \mathrm{~g})(\mathrm{P}>0.05)$. On the other hand, no difference $(\mathrm{P}>0.05)$ was observed between the average weights of the rabbits of the control batch N0 (59.06 g) and those of N5 and N15.

Keywords: Rabbit, Azadirachta indica, feeding, gestation, gestation rate

\section{Résumé}

La présente étude a été réalisée dans le cadre d'une série d'expérimentations visant l'utilisation des feuilles séchées pour la production des aliments complets destinés au lapin. A cet effet, 120 lapines mères, réparties en 4 lots (N0, N5, N10 et N15) de même effectif (30) et de poids moyens homogènes (2595 g à 2708,7 g) ont été suivies avant et pendant la gestation. Chaque lot était constitué d'autant de femelles nullipares que de femelles multipares. Les animaux ont été soumis à quatre aliments qui contenaient les feuilles de d'Azadirachta indica à différentes proportions : N0 : $0 \%$; N5 : $5 \%$; N10: $10 \%$ et N15: $15 \%$. Les taux de gestation se situaient comme suit pour les quatre lots : N0 (90\%), N5 (80\%) et N10 (90 $\%$ ) et N15 (76,67\%). A l'exception d'une femelle du lot N10, toutes les femelles déclarées gestantes ont effectivement mis bas. Aucune différence $(\mathrm{P}>0,05)$ n'a été notée entre les consommations journalières moyennes globales des lapines gestantes des quatre lots qui se situaient entre 169,40 et $198,31 \mathrm{~g} / \mathrm{j}$. Les tailles moyennes des portées des lots N0 (5,33 lapereaux/mère), N5 (6,5 lapereaux/mère) et $\mathrm{N} 10 \quad(5,61$ lapereaux/mère $)$ étaient semblables $(\mathrm{P}>0,05)$ mais plus élevées $(\mathrm{P}<0,05)$ que celle du lot $\mathrm{N} 15$ (4,46 lapereaux/mère). A la naissance, le poids moyen des lapereaux du lot N15 $(68,00 \mathrm{~g})$ a été significativement plus élevé $(\mathrm{P}<0,05)$ que ceux des lots 
N5 (48,63 g) et N10 (53,62 g) qui étaient similaires $(\mathrm{P}>0,05)$. Par contre, aucune différence $(\mathrm{P}>0,05)$ n'a été notée entre les poids moyens des lapereaux du lot témoin N0 (59,06 g) et ceux de N5 et N15.

Mots-Clefs: Lapin, Azadirachta indica, alimentation, gestation, taux de gestation

\section{Introduction}

La filière cunicole béninoise connaît ces dernières années un développement remarquable. Ce développement est, cependant, freiné par un certain nombre de facteurs dont le coût élevé de l'alimentation qui représente plus de $70 \%$ des charges de production des exploitations cunicoles (Soltner, 1994). De ce fait, les éleveurs et les acteurs du système national de la recherche scientifique sont en quête permanente de solutions alternatives susceptibles de contribuer à une baisse du coût des aliments. C'est dans ce cadre que, le Centre Technique d'Aviculture et d'élevage des petits animaux a entrepris depuis 2009 des études visant la valorisation des ressources alimentaires localement disponibles et accessibles à un coût réduit dans l'alimentation des lapins (Akoutey et Kpodékon, 2012 ; Kimse et al., 2013). Les études menées dans ce centre et par d'autres institutions de recherches et de formation sur la valorisation des fourrages déshydratées comme matière première pour la production des aliments complets destinés aux lapins ont donné des résultats assez satisfaisants qui permettent aujourd'hui de juger de l'intérêt alimentaire de certains fourrages (Moringa oleifera, Manihot esculenta, Leuceana leucocephala, Glyricidia seapium, Puerarea phaseoloides, Azadirachta indica.....) pour les animaux en engraissement (Adahê, 2007, Gbaguidi, 2011, Akoutey et Kodékon, 2012, Dahouda et al., 2013, Vidjannagni, 2013). Les résultats issus de ces essais laissent présager de la possibilité d'utiliser ces fourrages pour la production des aliments destinés aux reproducteurs. Cependant, au regard de la composition chimique de certains fourrages comme Azadirachta indica, leur valorisation chez les reproducteurs ne saurait être effective sans des essais préalables. En effet, les furano-tetranor terpenoids (azadirachtin, nimbidine, nimbine) et les molécules telles DNM-5 et DNM-7, contenus dans les feuilles de neem sont reconnues comme ayant des effets abortifs (Suryawanshi, 2011 et Formad environnement, 2013). De ce fait, il a été jugé, indispensable, d'étendre les essais menés sur les lapereaux en croissance aux femelles reproductrices. Cette étude dont le but était d'évaluer les effets des feuilles de neem, de la taille de la portée et de la parité sur la consommation alimentaire et les performances de production (fertilité, durée de gestation, taille de portée et poids des lapereaux à la naissance) a été réalisée sur les lapines vides et gestantes. 


\section{Matériel et méthodes}

\section{Site de l'expérimentation}

L'étude a été réalisée en 2014, entre juin et novembre, sur la ferme expérimentale du Centre Technique d'Aviculture et d'élevage des petits animaux. Cette ferme est installée dans l'un des 6 arrondissements de la commune de Tori-Bossito. Située entre $6^{\circ} 25^{\prime}$ et $6^{\circ} 37^{\prime}$ de latitude nord et $2^{\circ} 1^{\prime}$ et $2^{\circ} 17^{\prime}$ de longitude Est, cette commune jouit d'un climat subéquatorial caractérisé par deux saisons pluvieuses.

\section{Dispositif expérimental}

L'expérimentation a été menée sur 120 femelles de race locale, réparties en quatre lots de mêmes effectifs $(30 / \mathrm{lot})$, de même poids $(\mathrm{P}>0,05)$ et comprenant autant de femelles nullipares que de femelles multipares. Chaque lot de femelle était identifiable au taux d'incorporation de feuilles de neem $(\mathrm{FN})$ dans les aliments auxquels elles étaient soumise : N0 $(0 \% \mathrm{FN})$, N5 (5\% FN), N10 (10\% FN) et N15 (15\% FN). Les animaux ont été logés dans des cages grillagées de type mixte, de $0,34 \mathrm{~m}^{2}$. Ces cages étaient disposées en flat-desk sur des supports en bois (figure 1).

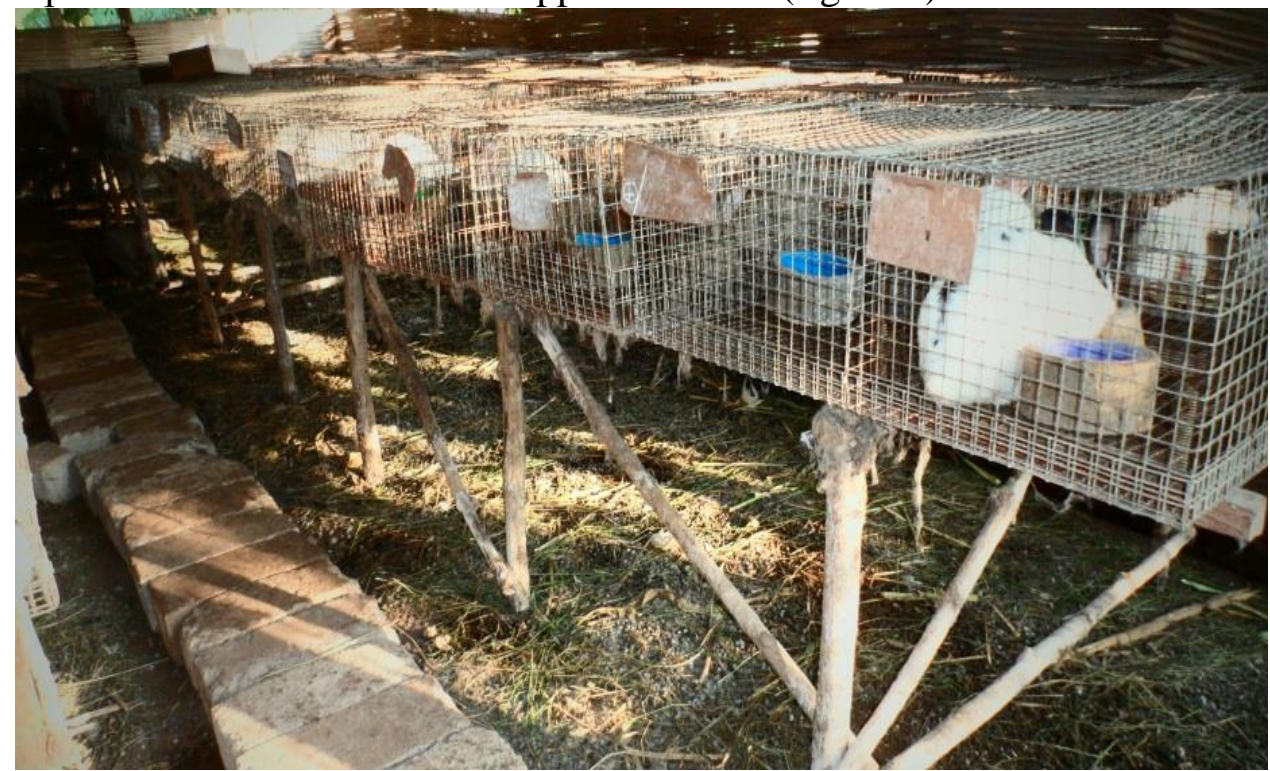

Figure 1. Vue interne du bâtiment d'élevage

Afin de réduire l'interférence des facteurs incontrôlés sur les résultats de l'étude, une randomisation des lapines des 4 lots a été réalisée dans la lapinière. La lapinière avait la forme d'un poulailler semi-plein air. Elle était protégée par des grillages et était ventilée et éclairée naturellement. Les mises en saillie des femelles ont été réalisées après détection visuelle des chaleurs (couleur rouge vive de la vulve). 
Soixante-douze heures avant la date présumée de mise bas, soit 13 jours après confirmation de la gestation par la palpation, il a été introduit dans les cages des femelles gestantes des boîtes à nid de dimensions $(\mathrm{L} * 1 * \mathrm{~h}): 40 \mathrm{~cm} * 25 \mathrm{~cm} * 12 \mathrm{~cm}$.

\section{Alimentation}

Les aliments avaient les mêmes valeurs protéiques et énergétiques (Tableau 1). Ils ont été formulé de manière à respecter les apports alimentaires recommandés pour les femelles reproductrices (Lebas et al., 2004). Les valeurs alimentaires des matières premières non testées ont été tirées des tables des valeurs alimentaires de Sauvant et al. (2004). La composition chimique des feuilles de neem est celle référencée par Djago et Kpodékon (2007) et Obun et al. (2013). Les valeurs nutritives des feuilles de neem $(\mathrm{PD}, \mathrm{ED})$ ont été considérée comme égales à celles des feuilles déshydratées de luzerne, tel que conseillé par Pesti et al. (2010).

Tableau 1. Compositions centésimales et valeurs alimentaires théoriques des aliments testés

\begin{tabular}{ccccc}
\hline & N0 & N5 & N10 & N15 \\
\hline & Composition centésimale (\%) & & \\
\hline Feuilles de neem & 15,00 & 5,00 & 10,00 & 15,00 \\
\hline Maïs & 2,00 & 7,50 & 2,00 & 0,00 \\
\hline Son de maïs & 19,75 & 28,00 & 23,00 & 19,50 \\
\hline Sonde Riz & 18,00 & 29,00 & 20,00 & 18,00 \\
\hline Tourteau de palmiste & 21,00 & 15,00 & 22,00 & 21,00 \\
\hline Tourteau coton & 11,00 & 5,00 & 9,50 & 11,00 \\
\hline Tourteau soja & 4,50 & 3,00 & 3,50 & 4,50 \\
\hline Farine de poisson & 4,00 & 3,00 & 4,00 & 4,00 \\
\hline Coquille & 2,00 & 2,00 & 2,00 & 2,00 \\
\hline Huile & 2,25 & 2,00 & 3,50 & 4,50 \\
\hline Sel & 0,20 & 0,20 & 0,20 & 0,20 \\
\hline Prémix & 0,30 & 0,30 & 0,30 & 0,30 \\
\hline
\end{tabular}

Valeurs alimentaires

\begin{tabular}{|ccccc|}
\hline Matière sèches & 89,62 & 89,35 & 89,62 & 89,48 \\
\hline Matières organiques (\%MS) & 175,22 & 170,70 & 165,60 & 161,37 \\
\hline Matières azotées totales (\%MS) & 18,46 & 16,49 & 18,85 & 19,88 \\
\hline Matières grasses totales (\%MS) & 3,96 & 3,82 & 3,70 & 3,61 \\
\hline Celluloses brute de weende (\%MS) & 11,81 & 11,22 & 12,57 & 12,63 \\
\hline Energie brute (Kcal/kgMS) & 3999,54 & 3975,24 & 4097,49 & 4116,09 \\
\hline Protéines digestibles (\%MS) & 12,92 & 12,84 & 12,98 & 13,29 \\
\hline
\end{tabular}


Les feuilles de neem utilisées dans le cadre de l'essai ont été collectées sur la ferme du CeTAvi situé au Nord-Est à encviron $35 \mathrm{Km}$ de Cotonou. Ces feuilles ont été déshydratées au soleil ( 2 à 3 jours) avant d'être combinées aux autres ingrédients alimentaires pour être moulus. Les aliments (farineux) et l'eau de boisson ont été servis deux fois par jour aux animaux (le matin : entre $7 \mathrm{~h}$ et $8 \mathrm{~h}$ et l'après-midi entre $16 \mathrm{~h}$ et $17 \mathrm{~h}$ ). La mise en consommation exclusive des aliments expérimentaux aux animaux, a été précédée d'une période de transition alimentaire de 20 jours au cours de laquelle, l'aliment usuel de la ferme a été progressivement remplacé par les aliments testés (plus $20 \%$ chaque 5 jours).

\section{Soins prophylactiques}

Au début de la phase de transition alimentaire, les animaux ont été déparasités avec 1'Alfamizol ${ }^{\circledR}$ (levamisol, Alfasan Holand, 1g/1,5L d'eau par jour) et d'anticox ${ }^{\circledR}$ (sulfamidine, Laprovet, France, 4g/20L d'eau pendant 3 jours). A la fin de ce traitement, les femelles ont été soumises à un traitement vitaminique $(5 \mathrm{~g} / 20 \mathrm{~L})$ de 3 jours à base d'Amin'total ${ }^{\circledR}$ (Laprovet, France). Le traitement vitaminique a été repris un mois plus tard.

\section{Collecte de données et analyses statistiques}

Les données ont été collectées sur les femelles vides et gestantes. Les chaleurs n'ayant pas été synchronisées, les données collectées sur les femelles vides ont duré de 10 à 25 jours (selon les femelles). Quant aux mesures effectuées sur les femelles gestantes, elles ont concerné toute la durée de gestation (30 à 34 jour selon les femelles). Au cours de celle-ci, il a été procédé, chez chaque femelle, aux pesées quotidiennes des quantités d'aliment servies et refusées, au relèvement de la durée de gestation, au comptage des lapereaux nés et à une pesée groupée de la portée. Les analyses statistiques ont été réalisées avec le logiciel SPSS (1.6). Les différents paramètres étudiés sont : l'ingestion alimentaire (ing), le taux de gestation (TG), le taux de mise bas (TMB), la durée de la gestation (dg), le nombre de lapereaux nés par lapine (tp) et le poids des lapereaux (P). Le taux de gestation ou taux de réussite de saillie est le pourcentage de femelles palpées positives par rapport à l'ensemble des femelles mises en reproduction. Quant au taux de mise bas, il représente la proportion (en \%) de femelles ayant mises bas par rapport aux femelles déclarées gestantes. Trois effets ont été étudiés : effet aliment, effet parité et effet classe de portée. Pour l'évaluation de l'effet classe de la portée les femelles ayant mis bas ont été répartis en trois classes $(\mathrm{C} 1, \mathrm{C} 2, \mathrm{C} 3)$ en fonction du nombre de lapereaux auxquels elles 
ont donné naissance : $\mathrm{C} 1$ : lapines ayant porté de 1 à 3 lapereaux, $\mathrm{C} 2$ : lapines ayant porté 4 à 6 lapereaux et $\mathrm{C} 3$ : lapine ayant des portées supérieures ou égales à 7 lapereaux. Une analyse de variance multivariée a été faite par la procédure des modèles linéaires généralisés (Proc GLM). Les moyennes des moindres carrés ont été estimées et comparées par le test de Duncan au seuil de probabilité de $5 \%$. Le test $X^{2}$ a été utilisé pour comparer les fréquences entre elles. Par ailleurs, il a été procédé à l'évaluation des corrélations ci-après : corrélation entre consommation alimentaire et corrélation entre la taille de la portée et poids moyens.

\section{Résultats}

\section{Ingestion alimentaire}

Aucune influence des trois effets étudiés n'a été perçue sur la consommation alimentaire des femelles vides. L'effet aliment a été observé sur la consommation alimentaire au cours des : $1^{\text {ère }}, 2^{\mathrm{e}}$ et $5^{\mathrm{e}}$ semaine de gestation $(\mathrm{P}<0,05)$. L'effet taille de la portée a uniquement été perçu sur la consommation alimentaire des lapines au cours de la deuxième semaine de gestation.

La Figure 2 compare les consommations alimentaires moyennes des femelles vides et gestantes des quatre lots expérimentaux. Dans tous les lots, la quantité d'aliment quotidiennement consommée par les femelles gestantes a été significativement plus élevée que celle des femelles non gravides $(\mathrm{P}<0,001)$. L'écart de consommation le plus élevé $(\mathrm{P}<0,05)$ a été constaté dans le lot N5 dont la consommation alimentaire moyenne des femelles gestantes a été augmentée de 99,23\% par rapport aux femelles vides. L'augmentation de consommation la moins élevée $(\mathrm{P}<0,05)$ a été obtenue dans le lot N15 $(55,04 \%)$.

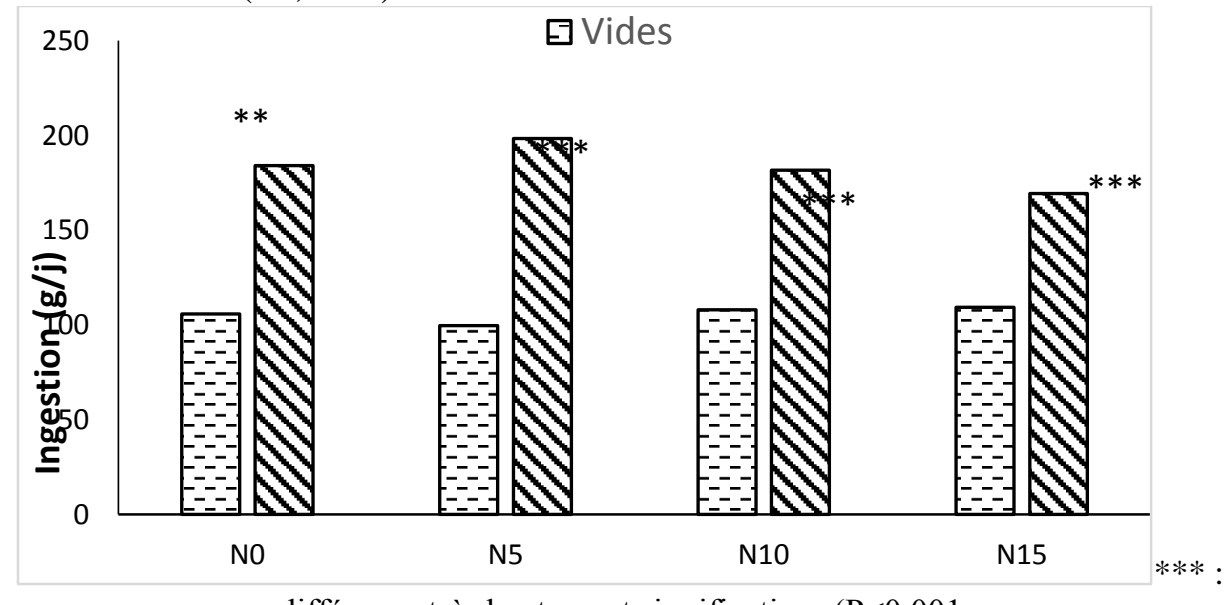

différence très hautement significatives $(\mathrm{P}<0,001$

Figure 2. Ingestions alimentaires moyennes des femelles vides et gestantes. 
L'effet aliment n'ayant pas été perçu sur l'ingestion alimentaire des lapines vides, les quantités journalières moyennes d'aliment $(99,63 \pm 6,80$ à $109,26 \pm 11,25 \mathrm{~g} / \mathrm{j}$ ) consommées par les femelles non gestantes des quatre lots ont été similaires $(\mathrm{P}>0,05)$ (Tableau 2).

Tableau 2. Ingestion alimentaire des lapines non gestantes et gestantes

\begin{tabular}{|c|c|c|c|c|c|c|c|c|c|c|c|c|}
\hline & \multicolumn{5}{|c|}{ Aliment } & \multicolumn{3}{|c|}{ Parité } & \multicolumn{4}{|c|}{ Classe de portée } \\
\hline & No & N5 & N10 & N15 & $\mathbf{P}<\mathbf{F}$ & Nul & Mult & $\mathbf{P}<\mathbf{F}$ & $\mathbf{C 1}$ & $\mathrm{C2}$ & $\mathrm{C3}$ & $\mathbf{P}<\mathbf{F}$ \\
\hline \multicolumn{13}{|c|}{ Ingestions des femelles non gestantes } \\
\hline Ing & $\begin{array}{l}105,86 \\
(8,32) \\
\end{array}$ & $\begin{array}{l}99,63 \\
(6,80) \\
\end{array}$ & $\begin{array}{l}108,02 \\
(9,45) \\
\end{array}$ & $\begin{array}{l}109,26 \\
(11,25) \\
\end{array}$ & ns & $\begin{array}{l}101,35 \\
(7,15) \\
\end{array}$ & $\begin{array}{l}102,08 \\
(6,88)\end{array}$ & ns & - & - & - & - \\
\hline \multicolumn{13}{|c|}{ Ingestions des femelles gestation } \\
\hline Ing $_{1-7}$ & $\begin{array}{l}182,60 \mathrm{a} \\
(11,23)\end{array}$ & $\begin{array}{l}149,1 \mathrm{~b} \\
(14,21)\end{array}$ & $\begin{array}{c}142,20 \mathrm{~b} \\
(12,03)\end{array}$ & $\begin{array}{c}151,20 b \\
(11,92)\end{array}$ & $*$ & $\begin{array}{c}164,50 \mathrm{a} \\
(8,41)\end{array}$ & $\begin{array}{c}149,40 \mathrm{a} \\
(8,98)\end{array}$ & ns & $\begin{array}{l}142,80 \\
(15,09)\end{array}$ & $\begin{array}{l}153,22 \\
(8,05)\end{array}$ & $\begin{array}{c}171,40 \\
(11,240\end{array}$ & ns \\
\hline Ing $_{7-14}$ & $\begin{array}{c}180,60 \mathrm{ab} \\
(12,45)\end{array}$ & $\begin{array}{c}209,30 \mathrm{a} \\
(15,75)\end{array}$ & $\begin{array}{c}174,80 \mathrm{ab} \\
(13,34)\end{array}$ & $\begin{array}{l}173,70 \mathrm{~b} \\
(13,22)\end{array}$ & $*$ & $\begin{array}{l}183,5 \\
(9,97)\end{array}$ & $\begin{array}{l}184,10 \\
(9,33)\end{array}$ & ns & $\begin{array}{l}155,70 \mathrm{a} \\
(16,72)\end{array}$ & $\begin{array}{c}190,88 \mathrm{~b} \\
(8,93)\end{array}$ & $\begin{array}{l}193,10 \mathrm{~b} \\
(12,37)\end{array}$ & $*$ \\
\hline $\operatorname{Ing}_{14-21}$ & $\begin{array}{l}184,20 \mathrm{a} \\
(13,69) \\
\end{array}$ & $\begin{array}{c}221,20 \mathrm{a} \\
(18,44) \\
\end{array}$ & $\begin{array}{l}174,70 \mathrm{a} \\
(11,02) \\
\end{array}$ & $\begin{array}{l}174,20 \mathrm{a} \\
(14,00) \\
\end{array}$ & ns & $\begin{array}{c}189,90 \mathrm{a} \\
(9,88) \\
\end{array}$ & $\begin{array}{l}175,00 \mathrm{a} \\
(10,51)\end{array}$ & ns & $\begin{array}{l}164,80 \mathrm{a} \\
(17,72) \\
\end{array}$ & $\begin{array}{c}184,41 \mathrm{a} \\
(9,46)\end{array}$ & $\begin{array}{l}191,50 \mathrm{a} \\
(13,10) \\
\end{array}$ & ns \\
\hline $\operatorname{Ing}_{21-28}$ & $\begin{array}{l}172,60 \mathrm{a} \\
(14,57) \\
\end{array}$ & $\begin{array}{l}198,00 \mathrm{a} \\
(16,69) \\
\end{array}$ & $\begin{array}{l}160,10 \mathrm{a} \\
(15,61) \\
\end{array}$ & $\begin{array}{l}161,20 \mathrm{a} \\
(15,47) \\
\end{array}$ & ns & $\begin{array}{l}172,30 \mathrm{a} \\
(10,91) \\
\end{array}$ & $\begin{array}{l}182,50 \mathrm{a} \\
(11,61) \\
\end{array}$ & ns & $\begin{array}{l}153,20 \mathrm{a} \\
(19,57) \\
\end{array}$ & $\begin{array}{l}190,46 a \\
(10,45) \\
\end{array}$ & $\begin{array}{l}176,20 \mathrm{a} \\
(15,48)\end{array}$ & ns \\
\hline $\begin{array}{c}\text { Ing }_{28-} \\
\text { 30à34 }\end{array}$ & $\begin{array}{c}183,60 \mathrm{~b} \\
(16,11) \\
\end{array}$ & $\begin{array}{c}240,30 \mathrm{a} \\
(20,40) \\
\end{array}$ & $\begin{array}{c}176,10 \mathrm{ab} \\
(17,11)\end{array}$ & $\begin{array}{c}186,50 \mathrm{ab} \\
(17,84)\end{array}$ & $*$ & $\begin{array}{l}189,40 \\
(12,07) \\
\end{array}$ & $\begin{array}{l}199,80 \\
(12,90) \\
\end{array}$ & ns & $\begin{array}{l}170,20 \mathrm{a} \\
(21,64)\end{array}$ & $\begin{array}{c}208,13 a \\
(11,56) \\
\end{array}$ & $\begin{array}{c}192,90 \mathrm{a} \\
(16,01)\end{array}$ & ns \\
\hline Ing 1-30à34 & $\begin{array}{c}184,12 \mathrm{a} \\
(8,53)\end{array}$ & $\begin{array}{c}198,31 \mathrm{a} \\
(9,76)\end{array}$ & $\begin{array}{c}181,69 \mathrm{a} \\
(8,79)\end{array}$ & $\begin{array}{l}169,40 \mathrm{a} \\
(11,12)\end{array}$ & ns & $\begin{array}{l}180,00 \\
(7,81) \\
\end{array}$ & $\begin{array}{l}178,00 \\
(8,34)\end{array}$ & ns & $\begin{array}{c}165,38 a \\
(12,21)\end{array}$ & $\begin{array}{c}187,81 \mathrm{a} \\
(6,21)\end{array}$ & $\begin{array}{c}189,88 \mathrm{a} \\
(8,64)\end{array}$ & ns \\
\hline
\end{tabular}

Ing : ingestion moyenne des femelles non gestantes, ing $\mathrm{i}-\mathrm{j}:$ ingestion sur la période $\mathrm{i}$ à $\mathrm{j}$ pendant la gestation Nul : Nullipare, Mult : multipares, C1 : taille de portée comprise entre 1 et $3, \mathbf{C 2}$ : taille de portée comprise entre 4 et $6, \mathrm{C} 3$ : taille de portée supérieure à $7, \mathbf{P}<\mathbf{F}$ : significativité, $\mathbf{a}, \mathbf{b}:$ les moyennes affectées de différentes lettres sont différentes au seuil de $5 \%, \mathbf{n s}$ : différences non significatif $(\mathrm{P}>0,05),{ }^{*}$ : différences significatives $(\mathrm{P}<0,05),{ }^{*}$ : différences significatives $(\mathrm{P}<0,05),()$ : Ecart-type

La Figure 3 présente l'évolution de la consommation alimentaire en fonction de la durée de la gestation. Cette figure révèle qu'en général, les consommations alimentaires des femelles gestantes, entre la première et la quatrième semaine, présentent une tendance générale à l'augmentation en fonction de l'âge de la gestation. Dans tous les lots les consommations alimentaires de la quatrième semaine de gestation ont été plus faibles que celles des troisième et cinquième semaines. 


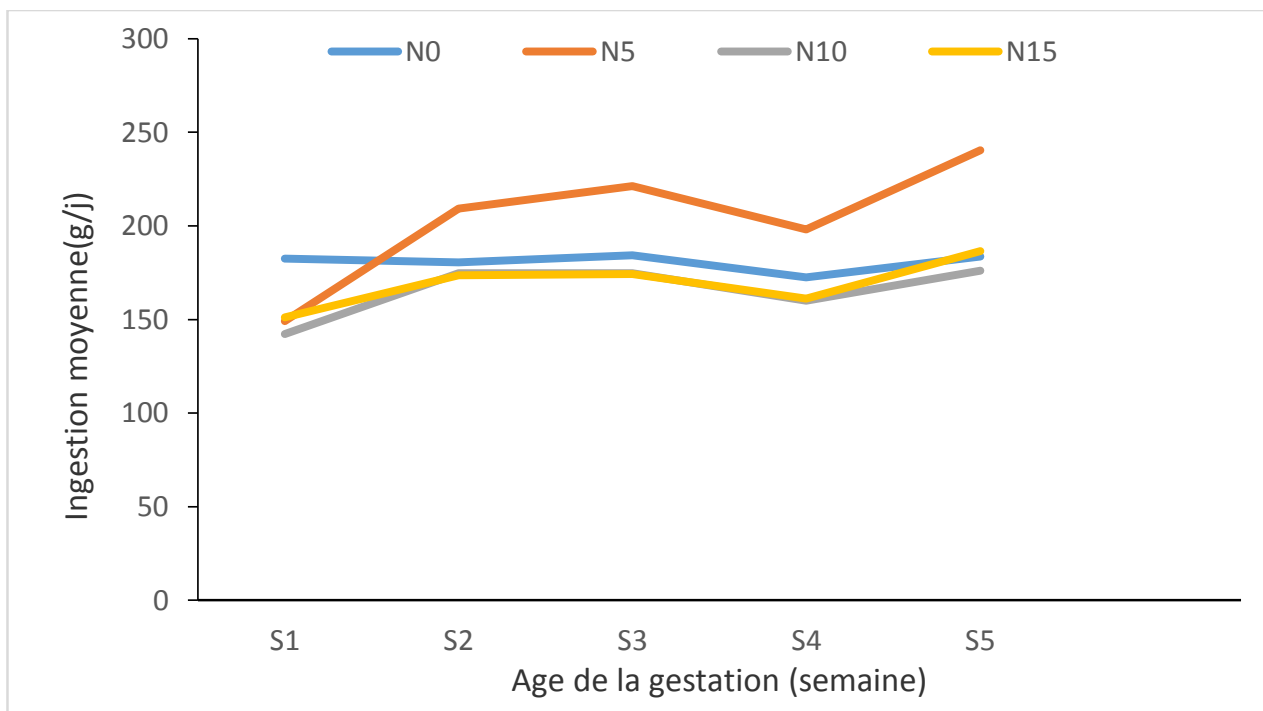

Figure 3. Evolution de la consommation alimentaire au cours de la gestation.

Les comparaisons des consommations alimentaires des femelles gestantes au cours de chaque période de gestation considérée ont révélé que pendant la première semaine de gestation, la consommation alimentaire des lapines du lot N0 $(182,60 \pm 12,51 \mathrm{~g} / \mathrm{j})$ a été plus élevée $(\mathrm{P}<0,05)$ que celles des lapines des trois autres lots qui se situaient entre $142,20 \pm 12,03 \mathrm{~g} / \mathrm{j}$ et $151,20 \pm 11,93 \mathrm{~g} / \mathrm{j}$ (Tableau 2). Par contre, dans la deuxième $\left(7^{\mathrm{e}}-14^{\mathrm{e}}\right.$ jour) et la cinquième semaine $\left(21^{\mathrm{e}}-28^{\mathrm{e}}\right.$ jour $)$ de gestation, c'est plutôt dans le lot N5 que les niveaux d'ingestions sont les plus élevés $(\mathrm{P}<0,05)$. Les ingestions alimentaires enregistrées dans les autres périodes de mesures considérées dans l'étude ont été identiques pour l'ensemble des quatre lots. Les consommations alimentaires moyennes des lapines des quatre lots durant toute la durée de la gestation (ing $\left.{ }_{1-30 / 34 \mathrm{j}}\right)$ étaient donc similaires $(\mathrm{P}>0,05)$ et se situaient entre $169,40 \pm 11,12 \mathrm{~g} / \mathrm{j}$ et $198,31 \pm 9,31 \mathrm{~g} / \mathrm{j}$ ).

Quel que soit les périodes de mesure, les quantités d'aliment ingéré par les femelles multipares n'ont pas été différentes de celles des femelles nullipares $(\mathrm{P}>0,05)$. La quantité moyenne d'aliment consommé par les femelles multipares au cours de la gestation a été évaluée à $178,00 \pm 8,34 \mathrm{~g} / \mathrm{j}$ contre $180 \pm 7,81 \mathrm{~g} / \mathrm{j}$ pour les nullipares.

Aucune différence $(\mathrm{P}>0,05) \quad$ n'a été observée entre les consommations alimentaires des femelles gestantes des trois classes de portées considérées dans le cadre de l'étude. Cependant, la tendance à l'augmentation de la consommation alimentaire en fonction de la taille de la portée, constatée sur toute la durée de la gestation, n'a été significative qu'au cours de la deuxième semaine. Au cours de cette semaine, les quantités d'aliment ingéré par les femelles appartenant aux classes de portée C2 
$(196,16 \mathrm{~g} / \mathrm{j})$ et C3 $(196,56 \mathrm{~g} / \mathrm{j})$, similaires $(\mathrm{P}>0,05)$ ont été plus élevées $(\mathrm{P}<0,05)$ que celles des femelles ayant porté de 1 à 3 lapereaux.

L'évaluation de la corrélation entre la taille de la portée et la consommation alimentaire des lapines gestantes a révélé une relation positive mais faible entre ces deux paramètres $(\mathrm{r}=0,31, \mathrm{P}<0,01)$.

\section{Performance de reproduction}

Le taux de gestation a été affecté par l'alimentation et la parité $(\mathrm{P}<0,05)$. Les résultats relatifs à la durée de la gestation (DG) et à la taille moyenne des portées (TMP) ont été significativement influencés $(\mathrm{P}<0,05)$ par l'ensemble des trois effets fixés (Tableau 3). Par contre l'effet de la parité n'a été constaté que sur le poids moyen des lapereaux à la naissance $(\mathrm{PMN})(\mathrm{P}<0,001)$.

Tableau 3. Effets des facteurs étudiés sur les performances de reproduction des lapines

\begin{tabular}{|c|c|c|c|c|c|c|c|c|c|c|c|c|}
\hline & \multicolumn{5}{|c|}{ Aliment } & \multicolumn{3}{|c|}{ Parité } & \multicolumn{4}{|c|}{ Classe de portée } \\
\hline & No & N5 & N10 & N15 & $\mathbf{P}<\mathbf{F}$ & Nullipare & Multipare & $\mathbf{P}<\mathbf{F}$ & C1 & $\mathrm{C2}$ & C3 & $\mathbf{P}<\mathbf{F}$ \\
\hline TG (\%) & $90 \mathrm{a}$ & $80 \mathrm{~b}$ & $90 \mathrm{a}$ & $76,67 b$ & $*$ & $75 \mathrm{~b}$ & $95 \mathrm{a}$ & $*$ & - & - & - & - \\
\hline TMB (\%) & 100 & 100 & 96,30 & 100 & ns & 98,33 & 100 & ns & & & & \\
\hline DG (jours) & $\begin{array}{c}31,80^{\mathrm{ab}} \\
(0,26)\end{array}$ & $\begin{array}{l}31,45^{b} \\
(0,33)\end{array}$ & $\begin{array}{c}31,79^{\mathrm{ab}} \\
(0,29)\end{array}$ & $\begin{array}{l}32,45^{\mathrm{a}} \\
(0,26)\end{array}$ & $*$ & $\begin{array}{l}31,87 \\
(0,19)\end{array}$ & $\begin{array}{l}31,97 \\
(0,20)\end{array}$ & ns & $\begin{array}{l}32,80^{\mathrm{a}} \\
(0,33)\end{array}$ & $\begin{array}{l}31,79^{\mathrm{b}} \\
(0,16)\end{array}$ & $\begin{array}{l}31,47^{\mathrm{b}} \\
(0,26)\end{array}$ & $* *$ \\
\hline $\begin{array}{l}\text { TMP } \\
\text { (lapereaux/ } \\
\text { mère) }\end{array}$ & $\begin{array}{l}4,48^{\mathrm{bc}} \\
(0,31)\end{array}$ & $\begin{array}{c}7,00^{\mathrm{a}} \\
(0,40)\end{array}$ & $\begin{array}{l}5,51^{\mathrm{b}} \\
(0,35)\end{array}$ & $\begin{array}{l}4,07^{\mathrm{c}} \\
(0,4)\end{array}$ & $* * *$ & $\begin{array}{c}5,26 \\
(0,23)\end{array}$ & $\begin{array}{c}4,88 \\
(0,24)\end{array}$ & ns & $\begin{array}{l}2,22^{\mathrm{c}} \\
(0,40)\end{array}$ & $\begin{array}{c}5,2^{\mathrm{b}} \\
(0,19)\end{array}$ & $\begin{array}{l}7,82^{\mathrm{a}} \\
(0,31)\end{array}$ & $* * *$ \\
\hline PMN (g) & $\begin{array}{l}59,06^{\mathrm{a}} \\
(2,34)\end{array}$ & $\begin{array}{c}48,63^{a b} \\
(2,91)\end{array}$ & $\begin{array}{l}53,62^{\mathrm{b}} \\
(2,82)\end{array}$ & $\begin{array}{l}68,00^{\mathrm{a}} \\
(2,84)\end{array}$ & $* *$ & $\begin{array}{l}52,00 \\
(1,87)\end{array}$ & $\begin{array}{l}66,42 \\
(1,94)\end{array}$ & $* * *$ & $\begin{array}{l}78,73^{\mathrm{a}} \\
(2,85)\end{array}$ & $\begin{array}{l}56,55^{\mathrm{b}} \\
(1,88)\end{array}$ & $\begin{array}{l}43,93^{b} \\
(2,62)\end{array}$ & $* * *$ \\
\hline
\end{tabular}

Nul : Nullipare, Mult : multipares, C1 : taille de portée comprise entre 1 et 3, C2 : taille de portée comprise entre 4 et $6, \mathrm{C} 3$ : taille de portée supérieure à $7, \mathbf{T G}$ : Taux de femelles gestantes ; TMB : taux de mise-bas ; DG : Durée moyenne de la gestation, TMP : taille moyenne des portées, $\mathbf{P M N}$ : Poids moyens à la naissance, $\mathbf{P}<\mathbf{F}:$ significativité, $\mathbf{a}, \mathbf{b}, \mathbf{c}:$ les moyennes affectées de différentes lettres sont différentes au seuil de $5 \%$, ns : différences non significatif $(\mathrm{P}>0,05), *$ : différences significatives $(\mathrm{P}<0,05), * *$ : différences hautement significatives $(\mathrm{P}<0,01), * * *$ : différences très hautement : $(\mathrm{P}<0,001)$,

\section{Taux de femelles gestantes (TG), Taux de Mise bas (TMB) et durées de gestation (DG)}

A l'issu de la palpation, il a été constaté que $82,75 \%$ des femelles mises en expérimentation ont été déclarées gestantes. Les meilleurs taux de gestation $(\mathrm{P}<0,05)$ ont été ceux des femelles des lots $\mathrm{N} 0(90,00 \%)$ et $\mathrm{N} 10$ $(90,00 \%)$. Le taux de gestation (TG) le plus bas a été obtenu avec le lot N15 $(76,67 \%)$. Le taux de gestation enregistré dans le groupe des femelles multipares $(95,00 \%)$ a été plus élevé $(\mathrm{P}<0,01)$ que celui des femelles multipares (75\%). Tous lots confondus, les mises-bas les plus précoces sont intervenues au $30^{\mathrm{e}}$ jour de gestation contre 34 jours pour les gestations les plus longues. La durée moyenne de gestation enregistrée dans le lot N15 a été plus longue que celle des femelles du lot N5 $(\mathrm{P}<0,05)$. Par contre, aucune 
différence n'a été notée entre les durées de gestation des femelles de ces lots avec celles des autres lots $(\mathrm{P}>0,05)$. Le temps moyen écoulé entre la saillie et la mise bas chez les femelles nullipares (31,87 jours) a été similaire $(\mathrm{P}>0,05)$ à celui des femelles multipares $(31,97$ jours $)$. La durée de gestation moyenne des femelles de la classe $\mathrm{C} 1$ (32,8 jours) a été plus élevée que celles des femelles des classes C2 $(31,79$ jours $)(\mathrm{P}<0,01)$ et $\mathrm{C} 3(31,35$ jours $)$ $(\mathrm{P}<0,001)$. Par contre, aucune différence n'a été observée entre la durée moyenne de gestation des femelles classées des classes C2 et C3.

En dehors d'une femelle du lot 10, aucun cas de fœtus avorté n'a été constaté durant 1'étude. De ce fait, les taux de mise bas enregistrés dans les quatre lots ont été identiques $(\mathrm{P}>0,05)$ pour les trois effets fixés.

\section{Taille de la portée et les poids des lapereaux à la naissance}

La femelle la plus prolifique, au cours de l'étude, a eu une taille de portée de 11 lapereaux contre 1 lapereau pour les femelles les moins prolifiques. Les tailles de portée les plus élevées ont été enregistrées dans le lot N5 (7,00 lapereaux/mère). Les tailles de portée les plus faibles $(\mathrm{P}<0,001)$ ont été enregistrées dans les lots N0 et N15 dont les TMP (4,48 et 4,05 lapereaux/mère) ont été similaires $(\mathrm{P}>0,05)$. La probabilité obtenue $(\mathrm{p}=0,003)$ sur 1'écart entre les TMP des lots N10 et N15 (1,44 lapereaux/mère) a permis de discriminer ces deux lots. Aucune différence $(\mathrm{P}>0,05)$ n'a été observée entre la prolificité des femelles nullipares $(5,26$ lapereaux/mère) et multipares (4,88 lapereaux/ mères).

A la naissance, les lapereaux du lot N15 pesaient en moyenne 68,00 \pm 2,84 g et étaient plus lourds $(\mathrm{P}<0,001))$ que les lapereaux du lot N5 dont le poids moyen $(52,45 \pm 2,91 \mathrm{~g})$ était inférieur $(\mathrm{P}<0,01)$ à celui du lot $\mathrm{N} 10$ $(57,73 \pm 2,82 \mathrm{~g})$. Le poids des lapereaux issus des femelles multipares $(66,42$ $\pm 2,06 \mathrm{~g})$ était plus élevé $(\mathrm{P}<0,001)$ que celui des femelles nullipares $(52,00$ $\pm 1,78 \mathrm{~g})$. Les meilleurs poids $(\mathrm{P}<0,001)$ à la naissance ont été enregistrés dans le groupe des femelles de classe de portée $\mathrm{C} 1$ (78,73 g vs 51,95 g et 44,09 g dans l'ordre pour les classes C2 et C3). Le poids moyen des lapereaux des femelles ayant des tailles de portée comprises entre 3 et 6 lapereaux ont également été plus élevés que celui des femelles du groupe $\mathrm{C} 3$. Au terme de l'analyse de corrélation, il a été établit une corrélation négative et forte $(\mathrm{r}=-0,68$ et $\mathrm{P}<0,001)$ entre la taille de portée et le poids moyens des lapereaux à la naissance.

\section{Discussion}

\section{Ingestions alimentaires}

Les résultats d'ingestion enregistrés au cours de l'étude montrent une indifférence générale des lapins aux aliments testés. Ainsi donc, contrairement aux prévisions, le goût amer des feuilles de neem n'a pas eu 
un effet répulsif sur la consommation alimentaire des lapines. Ce comportement alimentaire des lapins a été signalé par Gidenne et Lebas (2005) qui ont mentionné que les lapines ont une certaine préférence pour les aliments amers. Par ailleurs, à l'instar d'autres espèces fourragères ligneuses, les feuilles de neem renfermant des facteurs antinutritionnels (lignines, saponines, azadirachtine, oxalate,...), leur utilisation chez le lapin aurait pu induire une baisse de la consommation alimentaire si les doses tolérables étaient dépassées (Adjorlolo et al., 2016). A travers les constats faits au cours de cette étude, il peut être établit que, jusqu'à 15\% de taux d'incorporation dans l'aliment, la concentration des facteurs antinutritionnels contenus dans les feuilles n'affectent pas la consommation alimentaire des lapines reproductrices. Ces résultats sont en accord avec ceux obtenus par Wasanthakumar et al. (1999) qui ont montré que les lapins en engraissement peuvent supporter jusqu'à $20 \%$ de taux d'incorporation de tourteaux de neem dans la ration quotidienne.

Le niveau d'ingestion, plus élevé, enregistré chez les femelles gestantes (par comparaison aux femelles vides) doit être attribué à leur état physiologique. En effet, ces dernières, en plus de leur besoin individuel, doivent également satisfaire les besoins nutritionnels de leurs fœtus. A cet effet, Fortun-Lamothe et Lebas (1998) mentionnent que, pour satisfaire les besoins énergétiques plus élevées liées aux besoins énergétiques d'entretien des fotus, les lapines augmentent leur consommation alimentation durant la gestation. A ce propose, Parigini Bini (1990), signale qu'au cours de la gestation la consommation alimentaire des lapines s'accroit de 25 à $50 \%$ Les différences notées entre les consommations alimentaires des lapines au cours de la première, la deuxième et la cinquième semaine ne sauraient être liés à l'utilisation des feuilles de neem dans les lots N5, N10 et N15. En effet, excepté la première semaine où l'aliment témoin (dépourvu de feuille de neem) a été mieux ingéré, les niveaux d'ingestion des aliments à base de feuilles de neem ont été similaires (cas N10 et N15) ou supérieurs (cas N5) à ceux de l'aliment N0. Par contre, ces différences pourraient être associées à la taille de la portée (donc aux nombre de fotus portés par les lapines). A l'instar de l'étude menée par Amoussa (2011), il a été établit à la fin de l'essai qu'il existe une corrélation positive $(\mathrm{r}=+0,31)$ entre la taille de la portée et l'ingestion alimentaire des lapines. Le coefficient de corrélation obtenu ici a d'ailleurs été plus élevé que les 0,25 calculé par Amoussa (2011). Selon Parigini-Bini et Xiccato, (1998), le besoin alimentaire des lapines est fonction du poids de la portée (et donc indirectement de la taille de la portée). A cet effet, Amoussa (2011) a obtenu un coefficient de corrélation positif $(\mathrm{r}=+0,69)$ entre le poids de la portée et l'ingestion des lapines gestantes. 
La période de chute de consommation constatée au cours de la gestation $\left(21^{\mathrm{e}}\right.$ et $28^{\mathrm{e}}$ jour de gestation) coïncide avec la période de préparation de la boîte à nid par les femelles. Au cours de cette période où les lapines s'affairent à s'épiler et à tapisser la boîte à nid de poils et de fourrage, elles réduisent le temps consacré à l'alimentation. D'autres auteurs ont également noté une diminution progressive de la consommation en fin de gestation (Reyne et al., 1977, Gidenne et Lebas, 2005 et Amoussa, 2011). A l'instar du constat fait dans la présente étude, Reyne et al. (1977) ont observé que cette chute est plus prononcée pendant les 7 derniers jours de gestation et serait due aux perturbations physiologiques provoquées par la parturition.

Par ailleurs, les prises alimentaires enregistrées chez les lapines gestantes se situent dans la marge des valeurs consignées par Iyeghe et al. (2008) : 97,6 à $119 \mathrm{~g} / \mathrm{j}$. Elles sont également plus élevées que celles obtenues $(86,01$ à $161,63 \mathrm{~g} / \mathrm{j})$ par Amoussa (2011) et ce, malgré que les aliments testés par cet auteur se présentaient sous la forme de granulée.

\section{Performances de reproduction}

\section{Taux de gestation, taux de mise-bas et durée de gestation}

Le Taux de gestation globalement enregistré au cours de l'essai $(84,17 \%)$, de même que les TG obtenus dans les quatre lots, sont supérieurs à la moyenne $(70 \%)$ enregistrée dans les exploitations cunicoles des régions tropicales (Djago et Kpodékon, 2007). Les taux de femelles gestantes obtenus dans les lots N0 ; N5 et N10 sont également supérieurs à ceux des régions tempérées : 71,8\% à 78\% (Fromont et Tanguy, 2011). Quant à celui du lot N15 il s'écarte du meilleur taux mentionné par Fromont et Tanguy (2011) de $-3 \%$. Les taux de gestation obtenus dans les lots N0 et N10 se situent dans la marge des taux de gestation enregistrés dans les exploitations cunicoles béninoises par Akpo et al. (2008) : 85 à 94\%. Ce qui n'est pas le cas des TG des lots N5 et N15 qui sont plus faibles.

Dans une étude visant l'évaluation de l'effet des feuilles de Synedrella nodiflora sur les performances de reproduction des lapines, Koutinhouin et al. (2014) ont conclu que ces feuilles pourraient améliorer le taux de gestation des lapines. Le taux de fertilité enregistré par ces auteurs dans le lot témoin $(70 \%)$ est similaire aux taux de gestation des lots N5 et N10. Par contre le taux de fertilité enregistré dans le lot N15 est supérieur à celui du lot témoin de koutinhouin et al. (2014) mais est plus faible que ceux des lots de femelles ayant consommé les feuilles de $S$. nodiflora. Il peut donc être établit à partir de ces comparaisons, qu'à $5 \%$ de taux d'incorporation, l'effet des feuilles de neem sur la fertilité des lapines est similaire à celui des feuilles S.nodiflora lorsqu'elles sont uniquement consommées par les lapines. Par contre à $10 \%$ de taux d'incorporation dans l'aliment, l'effet de ces feuilles sur la fertilité des lapines est meilleur à celui de S. nodiflora. 
Le taux de gestation, plus faible, enregistré dans le lot N5 par rapport au lot N0, ne saurait être associé aux feuilles de neem. En effet, si c'était le cas, le taux de gestation du lot N10 aurait dû être plus bas que celui du lot N5. Par contre, le faible taux de gestation, constaté avec les femelles du lot $\mathrm{N} 15$, amène à penser qu'entre 10 et $15 \%$ de taux d'incorporation de feuilles séchées dans l'alimentation des lapines, un au moins des constituants à effet abortifs du neem (azadirachtine, nimbine, nimbidine, salannine, et les molécules DNM-5 et DNM-7) seraient suffisamment concentré pour entrainer des avortements. En effet, Suryawanshi (2011) signale que certains composés du neem peuvent provoquer des avortements chez certains mammifères (lapines, rate et singe) car ayant la capacité d'empêcher l'implantation des œufs fécondés dans l'utérus des femelles. Par ailleurs, il se pourrait également qu'entre 10 et $15 \%$ de taux d'incorporation les constituants chimiques des feuilles de neem aient bloqué l'ovulation chez certaines femelles (Delesalle, 2016).

Bien qu'aucun cas d'avortement n'ait été observé pendant l'étude, il peut être supposé que des pertes partielles ou totales de fœetus (mortalités embryonnaires), sont intervenues dans les lots N10 et N15 avant le jour de la palpation. A cet effet Suryawanshi (2011) signale que les constituants chimiques du neem stimulent le système immunitaire des femelles gestantes à réduire l'efficacité des hormones de croissances (EGF) des embryons au stade blastocyste. Or, ces hormones interviennent dans la différenciation et la survie cellulaire notamment au cours de l'embryogénèse (Barritault et al., 1982) en stimulant la mitose des cellules. Ce blocage de l'activité des facteurs de croissance épithéliale (EGF) au niveau des embryons, pendant la première semaine de gestion, cause la dégénérescence des embryons au stade blastocyste (Bhowmik, 2010, Suryawanshi, 2011 et Delesalle, 2016).

Lors de leurs études, Chrysostome et al., (2011) ont eu à remarquer que les lapines de race locale avaient une durée moyenne de gestation de 31,8 jours contre 31 jours pour les lapines de race Hyla. Les résultats obtenus dans les lots N0, N5 et N10 sont en accord avec les résultats obtenus par ces auteurs sur les femelles de races locales. La durée moyenne de gestation enregistrée dans le lot N15 est néanmoins plus longue que celle mentionnée par ces auteurs.

Le seul cas d'avortement observé après la palpation, ne permet pas d'attribuer l'avortement à l'utilisation des feuilles de neem dans la ration N10. Les taux de mise bas enregistrés dans l'étude sont semblables à ceux obtenus par Koutinhouin et al. (2014) avec les femelles ayant consommé $100 \%$ de feuilles de Synedrella nodiflora. 


\section{Taille de portée et poids moyens des lapereaux à la naissance}

Contrairement à ce qu'on aurait dû constater, la taille moyenne de portée enregistrée dans le lot N5 a été plus élevée que celle du lot témoin. Cette tendance à l'amélioration des performances de reproduction des lapines nourries aux aliments contenant $5 \%$ de feuilles de neem s'accorde parfaitement avec les résultats des études effectuées par Ogboewu (2008). En effet, ce dernier a eu à enregistrer une amélioration des paramètres morphométriques de l'appareil reproducteur des lapines lorsqu'elles sont soumises à un aliment contenant $5 \%$ de feuilles de neem.

Quel que soit le type d'aliment, les tailles de portée enregistrées au cours de l'étude sont inférieures à celles enregistrées par Koutinhouin et al. (2014) avec des femelles ayant consommé les feuilles de Synedrella nodiflora. La taille de portée la plus proche de ces valeurs est celle du lot N5. La taille moyenne de portée du lot N10 est assez voisine de celles des lots de femelles n'ayant pas consommé les feuilles de Synedrella nodiflora. La taille moyenne de portée des lapines des lots N0 et N15 sont plus faibles que celles obtenues dans des études antérieures (Koutinhouin et al., 2014 ; Amoussa, 2011 ; Akpo et al., 2008).

Au regard de la valeur du coefficient de corrélation obtenue entre le poids des lapereaux à la naissance et la taille des portées, l'on ne saurait attribuer les différences entre les poids des lapereaux à la naissance aux feuilles de neem. Mais plutôt à la taille des portées. Un tel constat a été fait par Amoussa (2011) qui a également constaté une corrélation très forte entre les poids des portées et la taille de la portée. Les poids individuels des lapereaux de cette étude $(48,62$ à $62,38 \mathrm{~g})$ sont assez voisins, de ceux (48 à $62 \mathrm{~g})$ obtenus par Berchiche (2012). Par contre ils sont en général meilleurs à ceux enregistrés (49,5 à 52,25 g) par Koutinhouin et al. (2014).

\section{Conclusion}

L'objectif de cette étude était d'évaluer la possibilité d'utiliser les feuilles de neem dans l'alimentation des lapines reproductrices vides et gestantes. Au regard des résultats relatifs à la consommation alimentaire des lapines, il ressort que, jusqu'à $15 \%$ de taux d'incorporation, les feuilles de neem n'influence pas l'ingestion alimentaire des lapines. Ces feuilles ont un effet bénéfique sur la taille de la portée de lapine lorsque leur taux d'incorporation dans l'aliment ne dépasse pas $5 \%$. Par contre, elles peuvent induire une baisse de productivité chez les lapines si elles sont introduites à plus de $10 \%$ dans la ration. L'étude a également révélé que la taille de la portée est le principal facteur qui influence le poids moyens des lapereaux à la naissance. En référence à l'ensemble des résultats enregistrés lors de l'étude, il peut être recommandé de ne pas incorporer plus de $5 \%$ de feuilles de neem dans l'alimentation des lapines vides et gestantes. La présente étude 
devra être étendue aux femelles allaitantes afin d'évaluer l'effet des feuilles sur la croissance pondérale des lapereaux, la viabilité des lapereaux et la santé des lapines.

\section{References:}

1. Adjorlolo, L. K., Timpong-Jones, E. C., Boadu, S. \& Adogla-Bessa, T. (2016). Potential contribution of neem (Azadirachta indica) leaves to dry season feeding of ruminants in West Africa. Livestock Research for Rural Development, 28 (5). www.lrrd.org/lrrd28/5/adjo28075.htm

2. Akoutey, A., \& Kpodékon, M. (2012). Performances zootechniques de lapereaux recevant des aliments granulés contenant du Pueraria phaseoloides. Tropicultura, 30 (3) : 161-166.

3. Akpo, Y., Kpodékon, T. M., Tanimomo, E., Djago, A. Y., Youssao A. K. I., Coudert, P. (2008). Evaluation of the reproductive performance of a local population of rabbits in south Benin. 9th World Rabbit Congress - June 10-13, 2008 - Verona - Italy. https://world-rabbit-science.com/WRSA-Proceedings/Congress2008-Verona/Papers/G-Akpo.pdf

4. Amoussa, S. (2011). Mesure de l'ingestion alimentaire volontaire et effet de l'association fourrage-concentré sur l'ingestion de la matière sèche chez les lapines vides, gestantes et allaitantes. Mémoire pour l'obtention du diplôme d'ingénieur agronome. FSA/UAC, Bénin, $85 \mathrm{p}$.

5. Barritault Moenner, M. \& Loret C. (1982). Nature et propriétés de divers facteurs de croissance. médecine/sciences. 1: 80-85. www.ipubli.inserm.fr/bitstream/handle/10608/3303/MS_1985_2_80. pdf? sequence $=1$

6. Berchiche, M., Cherfaoui, D., Lounaouci, G., \& Kadi, S. A. (2012). Utilisation de lapins de population locale en élevage rationnel : aperçu des performances de reproduction et de croissance en Algérie. $3^{\text {ème }}$ Congrès Franco-Maghrébin de zoologie et d'Ichtyologie. 6-10 novembre, Marrackech, Maroc.p.4.

7. Bhowmik, D., Chiranjib, Yadav J., Tripathi K. K. \& Kumar, K. K. S. (2010). Herbal remedies of Azadirachta indica and its Medicinal Application. Journal of Chemical and Pharmaceutical Research. 1: 62-72. Www.jocpr.com/articles/herbal-remedies-of-azadirachtaindica-and-its-medicinal-application.pdf

8. Chrysostome, C. A. A. M., Houndonougbo, M. F., Gbangboché, A. B. \& Houangni, M. S. M. (2011). Evaluation of reproduction performance of Hyla rabbits in hot and humid region in Benin. Research Opinion Animal Veterinary Sciences, 1(10): 669-672 
9. Dahouda, M., Adjolohoun, S., Sènou, M., Toleba, S. S., Abou, M., Vidjannagni, D. S., Kpodékon, M. \& Youssao A. K. I. (2013). Effets des aliments contenant les folioles de Moringa oleifera Lam et des aliments commerciaux sur les performances de croissance des lapins (Oryctolagus cuniculus) et la qualité de la viande. International Journal of Biological and Chemical Sciences, 7 (5): 1838-1856.

10. Delesalle, V. F. C. (2016). Ethno-pharmacognosie vétérinaire en élevage avicole, bovin, bubalin et porcin au Cambodge. Thèse pour l'obtention du doctorat vétérinaire. Ecole nationale vétérinaire d'Alfort, $\quad$ France. $321 \quad$ p. http://theses.vetalfort.fr/telecharger.php?id=2114

11. Djago, A. Y. \& Kpodékon, M. (2007). Méthodes et techniques d'élevage du lapin: élevage en milieu tropical. www.cuniculture.info/Docs/Elevage/ Tropic-01.htm

12. Formad environnement, (2013). Le margoussier ou neem. 18 p.

13. Fromont, A. \& Tanguy, M. (2011). L'élevage de lapins. Tom1. Educagri Editions, Dijon, France, 177p.

14. Fortun-Lamothe, L., \& Lebas, F. (1998). Effects of simultaneous pregnancy and lactation in primiparous rabbit does on weight and composition of new born rabbits. In: Rabbit production in hot climates (p. 10). Presented at 2. International conference, Adana, TUR (1998-09-07 1998-09-08). http://prodinra.inra.fr/record/137535.

15. Gbaguidi, J. A. V. (2011). Valorisation des sous-produits de soja et des feuilles de légumineuses (Moringa oleifera et Leuceana leucocephala) dans l'alimentation des lapereaux sevrés à Houenoussou dans la commune de Ouèssè-république du Bénin. Mémoire pour l'obtention du diplôme d'ingénieur des travaux agricoles. FSA/UAC, Bénin, 58 p.

16. Gidenne, T. \& Lebas, F. (2005). Le comportement alimentaire du lapin. 11èmes Journées de la Rechreche Cunicole, 29-30 novembre 2005, Paris, France.

17. Iyeghe-Erakpotobor, G.T., Adeosun, Y.G., Sekoni, A.A. \& Esievo, L.O. (2008). Reproductive performance of rabbit does on concentrate to forage (Stylosanthes hamata) combinations. Livestock Research for Rural Development, $20 \quad$ (11). WwW.1rrd.org/lrrd20/11/iyeg20178.htm

18. Kimse, M., Soro, D., Bléyéré, M. N., Yapi, J. N. \& Fantodji A. (2013). Apport d'un fourrage vert tropical, Centrosoma pubescens, en complément au granulé : effet sur les performances de croissance et sanitaire du lapin (Oryctolagus cuniculus). Internatinal Journal of 
Biological and Chemical Sciences, 7 (3): 1234-1242. http://ajol.info/index.php/ijbcs

19. Koutinhouin, G. B., Tougan, P. U., Kpodékon, T. M., Boko, K., C., Goudjihounde, M. Aoulou, A., \& Thewis, A. (2014). Valuation of Synedrella nodiflora leaves in rabbit feeding as feed supplement: impact on reproduction performance. International Journal of Agronomy and Agricultural Research, 5 (4): 55-64.

20. Ogboewu, I. P. (2008). Physiological responses of rabbits fed graded levels of neem (Azadirachta indica) leaf meal. Thesis submitted to the post-graduate school, Federal University of Technology, Owerr I. In partial fulfillment of the requirements for the award of the degree of Master of Science in animal reproductive physiology. 200p.

21. Parigi Bini, R. (1990). Repartions de l'énergie alimentaire chez la lapine non gestante pendant la première lactation. Proceedings des $5^{\text {èmes }}$ Journées de la Recherche Cunicole, France, Paris, 12-13 Décembre, Communication No.47.

22. Pesti, G., Thomsom, E., Bakalli, R., Leclercq, B., Shan, A., Atencio, A., Driver, J., Zier, C., Azain, M., Pavlack, M., Gidenne, T. \& Lebas, F. (2010). WUFFDA (version 1.4 adapté au lapin): logiciel de formulation alimentaire d'usage facile sous windows, guide d'utilisation-formulation.doc.

23. Reyne, Y., Prud'Hon, M., Debicki, AM., Goussopoulos, J., Angevain, J. (1977). Caractéristiques des consommations d'aliments solide et liquide chez la Lapine gestante puis allaitante nourrie. Annales de zootechnie, INRA/EDP Sciences, 1978, 27 (2): 211-223. https://hal.archives-ouvertes.fr/hal-00887803/document.

24. Soltner, D. (1994). Alimentation des Monogastriques. Sciences et Techniques Agricoles. Sainte -Gemmes -sur -Loire : Paris, France ; $84 \mathrm{p}$.

25. Suryawanshi, A. J. S. (2011). Neem-natural contraceptive for male and female - an overview. International Journal of Biomolecules and Biomedicine, $\quad 1 \quad$ (2): $\quad 1-6 \quad$ www.innspub.net/wpcontent/uploads/2012/01/IJBB-V1-No-2-p1-6.pdf

26. Vidjannagni, D. S. (2013). Pratiques d'élevage et d'alimentation de lapin au Bénin et perspectives d'amélioration à l'aide de ressources alimentaires non conventionnelles. Mémoire pour l'obtention du DEA en Management des Ressources Animales. FSA/UAC, Bénin. 194 p.

27. Wasanthakumar, P., Sharma, K., Sastry, V. R. B. \& Agrawal, D. K. (1999). Effect of_replacement peanut meal by neem (Azadirachta indica) seed kernel cake on_nutrient intake, digestibility and 
retention, and on body weight of broiler rabbits. World Rabbit Science, 7 (3):145-149. www.wrs.upv.es/files/journals/vol\%207_3 